[9] R. Cepeda-Gomez and N. Olgac, "Stability of the consensus of a group of second order agents with time delayed communications," in Proc. ASME Dyn. Syst. Control Conf., Boston, MA, 2010, pp. 361-368.

[10] R. Cepeda-Gomez and N. Olgac, "Consensus of a group of second order agents with switching communications topologies and time delay," in Proc. IEEE Conf. Decision Control, Atlanta, GA, 2010, pp. 5474-5479.

[11] Y. P. Tian and C. L. Liu, "Consensus of multi-agent systems with diverse input and communication delays," IEEE Transa. Autom. Control, vol. 53, no. 9, pp. 2122-2128, Aug. 2008.

[12] N. Olgac and R. Sipahi, "An exact method for the stability analysis of time-delayed linear time invariant systems," IEEE Trans. Autom. Control, vol. 47, no. 5, pp. 793-797, May 2002.

[13] N. Olgac and R. Sipahi, "Complete stability robustness of third order LTI multiple time delay systems," Automatica, vol. 41, pp. 1413-1422, 2005.

[14] N. Olgac and R. Sipahi, "An improved procedure in detecting the stability robustness of systems with uncertain delay," IEEE Trans Autom. Control, vol. 51, no. 7, pp. 1164-1165, Jul. 2006.

[15] E. W. Weisstein, "Kronecker Product," MathWorld, Tech. Rep., 2011 [Online]. Available: http://mathworld.wolfram.com/KroneckerProduct.html

[16] C. Godsil and G. Royle, Algebraic Graph Theory. New York: Springer, 2004

[17] I. V. Sergienko, E. F. Galba, and V. S. Deineka, "Limiting representations of weighted pseudoinverse matrices with positive definite weights. Problem regularization," Cybern. Syst. Anal., vol. 39, no. 6, pp. 816-830, 2003.

[18] N. Olgac and B. Holm-Hansen, "A novel active vibration absorption technique: The delayed resonator," J. Sound Vibr., vol. 176, pp. 93-104, 1994.

[19] E. W. Weisstein, "Descartes' Sign Rule," MathWorld, Tech. Rep., 2011 [Online]. Available: http://mathworld.wolfram.com/DescartesSignRule.html

[20] R. Cepeda-Gomez and N. Olgac, "Exhaustive stability analysis in a consensus system with time delay and irregular topologies," Int. J. Control, 2011, to be published.

\section{Robust Control of Linear Systems With Disturbances Bounded in a State Dependent Set}

\author{
Reza Ghaemi, Ilya V. Kolmanovsky, Fellow, IEEE, and \\ Jing Sun, Fellow, IEEE
}

\begin{abstract}
This technical note examines attractiveness and minimality of invariant sets for linear systems subject to additive disturbances confined in a state-dependent set. Existence of a minimal attractor is proved under the assumption that the state-dependent set, in which the disturbance is confined, is upper-semi-continuous. In many practical applications, the disturbance may evolve in a compact set while being generated by a dynamic process with a given model and bounded input. Our results for systems subject to general disturbances confined in state-dependent sets are applied to this case to prove the existence of a minimal robust invariant attractor.
\end{abstract}

Index Terms-Minimal robust invariant attractor (MRIA).

\section{INTRODUCTION}

Control problems for discrete-time systems subject to bounded additive disturbances have been considered extensively in the literature (see, e.g., [1], [2] and references therein). Control approaches based on application of reference governors [3], [4] and model predictive controllers [5], [6] with guaranteed stability and convergence properties have been developed. The minimal robust positive invariant set (mRPI), i.e., the set of states that can be reached from the origin under a bounded state disturbance, has been used in such control strategies as well as for synthesis of controllers for uncertain systems and for computing maximal robust positive invariant sets [7], [8]. In particular, the mRPI sets turn out to be attractors in robust model predictive control (MPC) with constraints tightening [9] and are essential in the synthesis of tube MPC [1] algorithms. Since the computation of mRPI sets is computationally prohibitive, the characterization and the computation of approximations to mRPI sets have been studied (see [10] and references there in.)

In all of the aforementioned approaches it is assumed that disturbances are confined to a given compact set and, at any time instant, allowed to take arbitrary values within the set. However, this assumption may be conservative in the case where the disturbance dynamics are known or can be estimated. A special and prevailing case is when the disturbances are generated by physical processes and as a result they have limited rates of variation. Another case is when disturbances can be modeled as an output of a dynamic system driven by a set-bounded signal. Finally, the disturbances may represent the effects of omitted nonlinearities of dynamic systems and their bounds may be state-dependent. Rate-bounded additive disturbances, as special cases of general disturbance dynamics, are considered in [12] where the rate bound

Manuscript received September 15, 2009; revised February 13, 2010, July 29, 2010, and November 21, 2010; accepted December 20, 2010. Date of publication April 07, 2011; date of current version July 07, 2011. This work was supported in part by NSF ECS-0501284 and ONR N00014-05-1-0537. Recommended by Associate Editor T. Zhou.

R. Ghaemi is with the Department of Mechanical Engineering, Massachusetts Institute of Technology, Cambridge, MA 02139 USA (e-mail: ghaemi@mit. edu).

I. V. Kolmanovsky is with the Department of Aerospace Engineering, The University of Michigan, Ann Arbor, MI 48109 USA (e-mail: ilya@ umich.edu).

J. Sun is with the Department of Naval Architecture and Marine Engineering, The University of Michigan, Ann Arbor, MI 48109 USA (e-mail: jingsun@umich.edu).

Color versions of one or more of the figures in this technical note are available online at http://ieeexplore.ieee.org.

Digital Object Identifier 10.1109/TAC.2011.2141330 
on the disturbance is used to calculate an approximation to maximal constraint admissible set that is less conservative than in the case when no rate bound is assumed. Since the disturbance at each time instance is constrained by previous values, it is expected that incorporating the previous values of disturbance in the controller may provide better control performance including a smaller minimal invariant set and a larger basin of attraction of this set.

Considering the dynamics of the system and disturbance, the invariant set analysis is concerned with the augmented system of state and disturbance where the disturbance is confined in a set which is dependent on the augmented state. It turns out that if the set containing disturbances is state dependent, then the minimal invariant set may not be an attractor and therefore may not be used in robust control synthesis and analysis. Hence, in this technical note we introduce the notion of minimal robust invariant attractor (MRIA) and we propose to use it in robust control design. Related but less general classes of disturbances were studied in [11] where these disturbances were assumed to be upper-bounded by a function of state, and attractive and positive invariant sets were considered.

In this technical note we consider a more general class of disturbances confined in a state-dependent set than in the prior literature. This class of disturbances includes the set of rate-bounded disturbances as a sub-class. The first contribution of the technical note is the analysis and characterization of the MRIA set for a linear system subject to an additive disturbance confined in a state-dependent and bounded set. In particular, we prove existence of a MRIA set and characterize it when the state-dependent set is upper semi-continuous. The second contribution of the technical note, built on the first one, is to establish the existence of a minimal attracting invariant set for the case when disturbances evolve within a compact set according to a specified linear dynamic model. The MRIA set is smaller if the disturbance model is considered compared to the case where only the boundedness of the additive disturbance is assumed. A numerical example is provided that shows the size of the minimal invariant attracting set is considerably different in the two cases. Smaller MRIA sets can lead to a larger region of attraction of robust MPC [1] designs and yield less conservatism in their response. Some of the results are reported in [20], where the computation of an approximation of a MRIA set is presented.

The technical note is organized as follows: in Section III, we establish the existence of MRIA set for linear systems subject to additive disturbance where the disturbance belongs to an upper-semi-continuous state dependent set. In Section IV, we introduce the system where the disturbance evolves inside a compact set according to a known disturbance model and show that it is a special case of the system described in Section III. Given the dynamics of the disturbance, we establish existence of a MRIA set in the lifted space of state and disturbance in Section V. In Section VI, a numerical example where it is shown that the MRIA set is considerably less conservative if the the dynamics of the disturbance are considered.

\section{BASIC DEFINITIONS AND NOTATIONS}

The sets of non-negative integers and reals are denoted by $\mathbb{N}$ and $R_{+}$, respectively, i,e, $\mathbb{N}:=\{0,1,2, \cdots\}$ and $\mathbb{R}_{+}:=\{x \in R: x \geq 0\}$. Given two sets $X \subset R^{n}$ and $Y \subset R^{n}$, the Minkowski set addition is defined by $X \oplus Y:=\{x+y: x \in X, y \in Y\}$. We write $x \oplus X$ instead of $\{x\} \oplus X$. Given a set $X$ and a real matrix $M$ of compatible dimension, we define $M X:=\{M x: x \in X\}$. Given a matrix $M \in$ $R^{n \times n}, \rho(M)$ denotes the largest absolute value of its eigenvalues. A family of non-empty compact subsets of $\mathbb{R}^{n}$ is denoted by $\operatorname{Com}\left(\mathbb{R}^{n}\right)$. For $X \subset \mathbb{R}^{n}, C l(X)$ denotes the closure of $X$ and $X^{c}$ denotes the complement of $X$. A function $\delta: \operatorname{Com}\left(\mathbb{R}^{n}\right) \times \operatorname{Com}\left(\mathbb{R}^{n}\right) \rightarrow \mathbb{R}$ is a Hemimetric on $\operatorname{Com}\left(\mathbb{R}^{n}\right)$ if $\delta(A, B) \leq \delta(A, C)+\delta(C, B)$ for all $A, B, C \in \operatorname{Com}\left(\mathbb{R}^{n}\right)$ and $\delta(A, A)=0$ for all $A \in \operatorname{Com}\left(\mathbb{R}^{n}\right)$. If $\delta$ is a Hemimetric, then an open ball centered at $X \in \operatorname{Com}\left(\mathbb{R}^{n}\right)$ and radius $\epsilon$ is denoted by $B_{\epsilon}(X):=\left\{Y \in \operatorname{Com}\left(\mathbb{R}^{n}\right) \mid \delta(X, Y)<\epsilon\right\}$.

In hemi-metric space $\left(\operatorname{Com}\left(\mathbb{R}^{n}\right), \delta\right)$, a set is open if it is a union of open balls, defined by hemi-metric $\delta$. The hemi-metrics $\delta_{l}$ and $\delta_{u}$ are defined as $\delta_{l}(X, Y):=\inf \{\epsilon>0 \mid X \subset$ $\left.B_{\epsilon}(0) \oplus Y\right\}$ and $\delta_{u}(X, Y):=\delta_{l}(Y, X)$, respectively. The Hausdorff distance of two sets $A, B \subset \mathbb{R}^{n}$ is a metric given by $\delta_{H}(A, B)=\max \left\{\delta_{l}(A, B), \delta_{u}(A, B)\right\}$. A multi valued function $f: \mathbb{R}^{n} \rightarrow \operatorname{Com}\left(\mathbb{R}^{n}\right)$ is upper semi-continuous/lower semi-continuous if for any open set $A$ in hemi-metric space $\left(\operatorname{Com}\left(\mathbb{R}^{n}\right), \delta_{u}\right) /\left(\operatorname{Com}\left(\mathbb{R}^{n}\right), \delta_{l}\right), f^{-1}(A):=\left\{x \in \mathbb{R}^{n} \mid f(x) \in A\right\}$ is open in $\mathbb{R}^{n}$. A multi valued function is continuous if it is upper semi-continuous and lower semi-continuous, i.e., it is continuous with respect to space $\operatorname{Com}\left(\mathbb{R}^{n}\right)$ and metric $\delta_{H}$.

The Graph of a multi-valued function $f: \mathbb{X} \subset \mathbb{R}^{n} \rightarrow \operatorname{Com}\left(\mathbb{R}^{n}\right)$ is $G r f:=\{(x, y) \mid x \in \mathbb{X}, y \in f(x)\}$. A set valued function is called correspondence. For a correspondence $f$, strong pre-image of a set $A \subset$ $\mathbb{R}^{n}$ is $f^{s}(A):=\left\{x \in \mathbb{R}^{n} \mid f(x) \subset A\right\}$. For a set sequence $\left\{A_{i}\right\}$, $i \in \mathbb{N}, \limsup _{i \rightarrow \infty} A_{i}=\bigcap_{n=1}^{\infty} \bigcup_{i=n}^{\infty} A_{i}[15]$.

Pontryagin difference [7] of two sets $S$ and $T$ is defined as $S \sim T=$ $\{x \mid x+t \in S, \forall t \in T\}$.

\section{Characterization OF MRIA Sets: The General CASE}

Consider a discrete-time linear time-invariant system

$$
z^{+}=A z+\omega, \quad \omega \in \mathcal{W}(z)
$$

where $z \in \mathbb{R}^{r}$ is the current state, $z^{+}$is the successor state and $\omega \in$ $\mathbb{R}^{r}$ is an unknown disturbance taking values in a state dependent set $\mathcal{W}(z)$ and $\mathcal{W}: \mathbb{R}^{r} \rightarrow \operatorname{Com}\left(\mathbb{R}^{r}\right)$. In this technical note we make the following assumptions:

Assumption 1: The state transition matrix $A$ is strictly stable, i.e., $\rho(A)<1$.

Assumption 2: The multi-valued function $\mathcal{W}$ is upper semi-continuous.

Assumption 3: There exists a compact set $\mathbb{W} \subset \mathbb{R}^{r}$ such that for all $z \in \mathbb{R}^{r}, \mathcal{W}(z) \subseteq \mathbb{W}$.

The following Lemma provides another interpretation of upper semicontinuity, which is introduced in the Definition Section, [15].

Lemma 2.1: A correspondence $f$ is upper semi-continuous if for every open set $G$ in $\mathbb{R}^{r}$, the pre-image $f^{s}(G)$ is open in $\mathbb{R}^{r}$.

We recall the following standard definition [2]

Definition 2.1: A set $\Omega$ is an invariant set for the system (1) if for all $z \in \Omega, A z \oplus \mathcal{W}(z) \subseteq \Omega$.

Definition 2.2: A nonempty set $\Omega$ is an attractor for system (1), if for all initial conditions $z(0) \in \mathbb{R}^{r}$, any solution $z(k)$ with dynamics $z(k)=A z(k-1)+\omega, \omega \in \mathcal{W}(z(k-1))$ converges to $\Omega$ as $k \rightarrow \infty$. A set is a minimal attractor if it is contained in any attractor of the system.

In this technical note, we establish existence of the MRIA set for the system (1) under the assumptions 1-3.

Consider the map, induced by the set valued function $\mathcal{W}, \mathcal{R}$ : $\operatorname{Com}\left(\mathbb{R}^{r}\right) \rightarrow 2^{\mathbb{R}^{r}}$ given by

$$
\mathcal{R}(Z):=\{A z+\omega: z \in Z, \quad \omega \in \mathcal{W}(z)\}
$$

where $2^{\mathbb{R}^{r}}$ denotes the set of all subsets of $\mathbb{R}^{r}$. We denote by $\mathcal{R}^{j}(\cdot)$ the $j$-th iterate of the map $\mathcal{R}(\cdot)$, given by $(2)$, and $\mathcal{R}^{0}(Z)=Z$ for $Z \subset \mathbb{R}^{r}$. If $z \in \mathbb{R}^{r}, \mathcal{R}(z):=\{A z+\omega: \omega \in \mathcal{W}(z)\}$. Thus a set $\Omega \subset \mathbb{R}^{r}$ is invariant if and only if $\mathcal{R}(\Omega) \subseteq \Omega$. A set $\Omega \subset \mathbb{R}^{r}$ is an attractor if and only if for all $Z \in \operatorname{Com}\left(\mathbb{R}^{r}\right), \lim \sup _{j \rightarrow \infty} \mathcal{R}^{j}(Z) \subseteq \Omega$.

Lemma 2.2: For any $Z \in \operatorname{Com}\left(\mathbb{R}^{r}\right), \mathcal{R}(Z)$ is compact.

Proof: Since $\mathcal{W}: Z \rightarrow \operatorname{Com}\left(\mathbb{R}^{r}\right)$ is upper semi-continuous, so is $\mathcal{R}: Z \rightarrow \operatorname{Com}\left(\mathbb{R}^{r}\right)$ given by $\mathcal{R}(z)=A z+\mathcal{W}(z)$. Moreover, $\mathcal{R}$ 
is compact valued and given that $Z$ is compact, $\mathcal{R}(Z)=\bigcup_{z \in Z} \mathcal{R}(z)$ is compact (see [15, p. 90]).

Therefore, $\mathcal{R}(\cdot)$ maps $\operatorname{Com}\left(\mathbb{R}^{r}\right)$ to itself.

Lemma 2.3: Assume Assumptions 1 and 3 hold. Then the set

$$
F:=C l\left(\bigoplus_{i=0}^{\infty} A^{i} \mathbb{W}\right)
$$

is an attracting invariant set, i.e., $\mathcal{R}(F) \subseteq F$ and for any $K \in \operatorname{Com}\left(\mathbb{R}^{r}\right), \limsup \sup _{j \rightarrow \infty} \mathcal{R}^{j}(K) \subseteq F$.

Proof: The Lemma follows from Assumption 3 and the fact that $F$ is an attracting invariant set for the system $z^{+}=A z+\omega, \omega \in \mathbb{W}$ [13].

Lemma 2.4: Suppose $\left\{Z_{i}\right\}, i \in \mathbb{N}$ is a sequence of non-empty compact sets in $\mathbb{R}^{r}$ where $Z_{i+1} \subseteq Z_{i}$ for $i \in \mathbb{N}$. Then $\bar{Z}:=\bigcap_{i=1}^{\infty} Z_{i} \neq \emptyset$. Moreover, for all open sets $G \subset \mathbb{R}^{r}$ such that $\bar{Z} \subset G$, there exists $M \in \mathbb{N}$ such that $Z_{M} \subset G$.

Proof: See [17] for the proof.

Theorem 2.1: Suppose Assumptions 1-3 hold and $F$ is defined by (3). Then

$$
\Omega^{*}:=\bigcap_{j=0}^{\infty} \mathcal{R}^{j}(F)
$$

is non-empty and compact, i.e., $\Omega^{*} \in \operatorname{Com}\left(\mathbb{R}^{r}\right)$, and

$$
\mathcal{R}\left(\Omega^{*}\right)=\Omega^{*}
$$

Proof: The proof is similar to the one given for Theorem 8.2.1 in [15]. According to Lemma 2.3, $\mathcal{R}(F) \subseteq F$. Therefore, $\mathcal{R}^{j}(F) \subseteq$ $\mathcal{R}^{j-1}(F)$ for $j \in \mathbb{N}$. Since $\mathcal{W}(z) \neq \emptyset$ for all $z \in \mathbb{R}^{r}, \mathcal{R}^{j}(F) \neq \emptyset$. Hence, according to Lemma $2.4, \Omega^{*}=\bigcap_{j=0}^{\infty} \mathcal{R}^{j}(F) \neq \emptyset$. If $z \in \Omega^{*}$, then for all $n>0, z \in \mathcal{R}^{n}(F)$. Hence, $\mathcal{R}(z) \subset \mathcal{R}^{n+1}(F), n>0$. Therefore, $\mathcal{R}(z) \subset \Omega^{*}$ and $\mathcal{R}\left(\Omega^{*}\right) \subseteq \Omega^{*}$. Now we need to prove the reverse inclusion. Assume $z_{0} \in \Omega^{*} \backslash \mathcal{R}\left(\Omega^{*}\right)$. Then $\mathcal{R}\left(\Omega^{*}\right)$ and $z_{0}$ can be separated by disjoint open sets $U_{1}$ and $U_{2}$. Since $\mathcal{R}(\cdot)$ is upper semi-continuous, $\mathcal{R}^{s}\left(U_{1}\right)$ is open and since $\mathcal{R}\left(\Omega^{*}\right) \subset U_{1}$, we have $\Omega^{*} \subset \mathcal{R}^{s}\left(U_{1}\right)$. Therefore, according to Lemma 2.4, there exists $M \in$ $\mathbb{N}$ such that $\mathcal{R}^{M}(F) \subset \mathcal{R}^{s}\left(U_{1}\right)$. Hence, $\Omega^{*} \subset \mathcal{R}^{M+1}(F) \subset U_{1}$ and $z_{0} \in \Omega^{*} \subset U_{1}$ which contradicts $z_{0} \in U_{2}$. Therefore, $\Omega^{*} \backslash \mathcal{R}\left(\Omega^{*}\right)=\emptyset$ and $\mathcal{R}\left(\Omega^{*}\right)=\Omega^{*}$.

Theorem 2.2: Suppose Assumptions 1-3 hold. Then $\Omega^{*} \in$ $\operatorname{Com}\left(\mathbb{R}^{r}\right)$, defined in (4), is a minimal robust attractor with the basin of attraction being the whole space, $\operatorname{Com}\left(\mathbb{R}^{r}\right)$.

Proof: Given $K \in \operatorname{Com}\left(\mathbb{R}^{r}\right)$, according to Lemma 2.3

$$
\forall \epsilon>0, \exists M_{1} \in \mathbb{N} \text {, s.t } \forall n \geq M_{1}, \mathcal{R}^{n}(K) \subset F \oplus B_{\epsilon}(0)
$$

where $B_{\epsilon}\left(z_{0}\right):=\left\{z \in \mathbb{R}^{r} \mid\left\|z-z_{0}\right\|<\epsilon\right\}$. For any set $S \in \operatorname{Com}\left(\mathbb{R}^{r}\right)$, $S \oplus B_{\epsilon}(0)=\bigcup_{z \in S} B_{\epsilon}(z)$. Therefore, $S \oplus B_{\epsilon}(0)$ is an open set. From Assumption $2, \mathcal{R}(\cdot)$ is upper semi-continuous. Hence, according to Lemma 2.1, $\mathcal{R}^{s}\left(\mathcal{R}(S) \oplus B_{\epsilon}(0)\right)$ is open. Since the set $F$, defined in (3), is compact and contained in the open set $\mathcal{R}^{s}\left(\mathcal{R}(F) \oplus B_{\epsilon}(0)\right.$ ), for $\epsilon>0$, there exists $\delta(\epsilon)$ such that $F \oplus B_{\delta(\epsilon)}(0) \subset \mathcal{R}^{s}\left(\mathcal{R}(F) \oplus B_{\epsilon}(0)\right)$. Hence, for all $\epsilon>0$, there exists $\delta(\epsilon)>0$ such that

$$
\mathcal{R}\left(F \oplus B_{\delta(\epsilon)}(0)\right) \subset \mathcal{R}(F) \oplus B_{\epsilon}(0)
$$

According to Lemma 2.4 and (4), given $\epsilon>0$, there exists $M_{2}(\epsilon)>0$ such that

$$
\mathcal{R}^{M_{2}}(F) \subset \Omega^{*} \oplus B_{\epsilon / 2}(0) .
$$

Considering (7), we have $\mathcal{R}\left(\mathcal{R}^{M_{2}-1}(F) \oplus B_{\delta(\epsilon / 2)}(0)\right) \subset \mathcal{R}^{M_{2}}(F) \oplus$ $B_{\epsilon / 2}(0)$. Applying (7) recursively, it is deduced

$$
\mathcal{R}^{M_{2}}\left(F \oplus B_{\delta M_{2(\epsilon / 2)}}(0)\right) \subset \mathcal{R}^{M_{2}}(F) \oplus B_{\epsilon / 2}(0)
$$

where $\delta^{i+1}(\epsilon / 2):=\delta^{i}(\epsilon / 2)$ and $\delta^{0}(\epsilon / 2)=\epsilon / 2$. From (8) and (9) we have

$$
\mathcal{R}^{M_{2}}\left(F \oplus B_{\delta^{M_{2}(\epsilon / 2)}}(0)\right) \subset \Omega^{*} \oplus B_{\epsilon}(0) .
$$

According to (6), if $\left.\hat{\delta}:=\delta^{M_{2}}(\epsilon / 2)\right)$, there exists $M_{1}(\hat{\delta}) \in \mathbb{N}$ such that for all $n>M_{1}(\hat{\delta})$

$$
\mathcal{R}^{n}(K) \subset F \oplus B_{\hat{\delta}}(0) .
$$

From (11), for all $n>M_{1}+M_{2}$, we have $\mathcal{R}^{n}(K)=$ $\mathcal{R}^{M_{2}}\left(\mathcal{R}^{n-M_{2}}(K)\right) \subset \mathcal{R}^{M_{2}}\left(F \oplus B_{\hat{\delta}}(0)\right)$ and from $(10), \mathcal{R}^{M_{2}}(F \oplus$ $\left.B_{\hat{\delta}}(0)\right) \subset \Omega^{*} \oplus B_{\epsilon}(0)$. Therefore, for all $n>\left(M_{1}+M_{2}\right)(\epsilon)$, $\mathcal{R}^{n}(K) \subset \Omega^{*} \oplus B_{\epsilon}(0)$. Hence, $\lim \sup _{n \rightarrow \infty} \mathcal{R}^{n}(K) \subseteq \Omega^{*}$. Therefore $\Omega^{*}$ is an attractor. Assume $\Omega$ is another attractor. Then $\limsup \sup _{n \rightarrow \infty} \mathcal{R}^{n}\left(\Omega^{*}\right) \subseteq \Omega$. Since $R\left(\Omega^{*}\right)=\Omega^{*}, \Omega^{*} \subset \Omega$.

Remark 2.1: The set $\Omega^{*}$ is the fixed point of mapping $\mathcal{R}(\cdot)$ and according to Theorem 2.2 is the minimal robust invariant attractor, where invariance is defined in Definition 2.1. That is, the set $\Omega^{*}$, attracts all state trajectories, and any state trajectory with initial state inside the set, remains inside the set. Hence, it is unique regardless of choice of $W$ and the corresponding $F$.

In the sequel, as a special case, we consider existence and uniqueness of minimal invariant sets for linear systems subject to constraints and additive bounded disturbance that evolve according to a given dynamic model.

\section{Linear Systems With DistuRbanCE MODELS}

In this section, we formulate the problem where the disturbance evolves inside a compact set according to a known disturbance model. We consider a discrete-time linear system

$$
x^{+}=A x+B u+C w
$$

where $x \in \mathbb{R}^{n}$ is the current state, $u \in \mathbb{R}^{m}$ is the current control, $w \in \mathbb{R}^{p}$ is the disturbance, $x^{+} \in \mathbb{R}^{n}$ is the successor state and matrices $A, B$ and $C$ are of compatible dimensions.

\section{A. Disturbance Models}

In this subsection, we first consider the case where the disturbance is rate-bounded and formulate the disturbance model accordingly. Next we consider a more general case where the disturbance model evolves according to a specified dynamic equation inside a given compact set.

1) Magnitude and Rate-Bounded Disturbances: For a rate-bounded disturbance, we can assume that disturbance variation is confined in a compact set $V \in \operatorname{Com}\left(\mathbb{R}^{l}\right)$, i.e., $w-w^{-} \in V$, where $w \in \mathbb{R}^{p}$ is the current disturbance and $w^{-} \in \mathbb{R}^{p}$ is the predecessor disturbance. Therefore, the disturbance dynamics can be equivalently expressed as follows:

$$
w=w^{-}+v^{-}, \quad v^{-} \in V
$$

where $v^{-} \in \mathbb{R}^{l}$ is the predecessor disturbance model input.

While the disturbances evolve according to dynamic equation (13), their values are confined to the compact set $W$. Hence, the disturbance model state and input are subject to the following constraints:

$$
w \in W, \quad w^{-} \in W \quad \text { and } \quad v^{-} \in V
$$

where $W \in \operatorname{Com}\left(\mathbb{R}^{p}\right)$. 
2) General Model of Disturbance: A more general class of disturbance models can be expressed as follows:

$$
w=F w^{-}+G v^{-}
$$

where $w, w^{-}$and $v^{-}$are subject to the constraints (14).

Remark 3.1: In practical applications, the disturbance model (15), (14) can be constructed using system identification techniques applied to disturbance measurements, see e.g. [14].

\section{B. Augmented Linear System}

We now demonstrate that (12)-(14) can be presented as a linear system with additive disturbances confined in a state-dependent set.

The constraint (14) induces:

$$
\begin{aligned}
w & \in\left\{F w^{-}+G v^{-} \mid v^{-} \in V, \quad F w^{-}+G v^{-} \in W\right\} \\
& =\left(F w^{-}+G V\right) \cap W .
\end{aligned}
$$

We define a correspondence $g: W \rightarrow \operatorname{Com}(W)$ as

$$
g(w):=(F w+G V) \cap W .
$$

Note that (13) and (14) imply

$$
w \in g\left(w^{-}\right), \quad w^{-} \in W .
$$

Clearly for all $w \in W, g(w) \neq \emptyset$. It is assumed that at each time instant, the current state $x$ and the predecessor disturbance $w^{-}$are accessible to the controller but $v^{-}$or the current value of disturbance, $w$, are not. Given the known state and disturbances $x$ and $w^{-}$at each time instant, we assume that the controller applies a linear feedback given by:

$$
u=L x+M w^{-}
$$

where $L$ is chosen such that the matrix $A_{L}:=A+B L$ is strictly stable.

Remark 3.2: If the predecessor disturbance $w^{-}$is not available, then the matrix gain $M=0$. In the sequel, we include disturbance feedback in the control feedback law to cover both cases where the predecessor additive disturbance is known or unknown.

Incorporating the control law (19), the system and disturbance dynamics (12) and (13) subject to disturbance constraints (14) take the following closed-loop form:

$$
\left(\begin{array}{c}
x \\
w^{-}
\end{array}\right)^{+}=\left(\begin{array}{cc}
A+B L & B M \\
0 & 0
\end{array}\right)\left(\begin{array}{c}
x \\
w^{-}
\end{array}\right)+\left(\begin{array}{l}
C \\
I
\end{array}\right) w
$$

subject to constraints:

$$
w \in g\left(w^{-}\right) .
$$

Introducing the augmented state $z=\left(x, w^{-}\right),(20)$ takes the following form:

$$
z^{+}=\tilde{A} z+\omega, \quad \omega \in \mathcal{W}(z)
$$

where $\mathcal{W}: \mathbb{R}^{n} \times W \rightarrow \mathbb{R}^{n} \times W$ is given by

$$
\mathcal{W}(z):=\tilde{B} g([0 \Gamma] z)
$$

and

$$
\tilde{A}:=\left(\begin{array}{cc}
A+B L & B M \\
0 & 0
\end{array}\right), \quad \tilde{B}:=\left(\begin{array}{l}
C \\
I
\end{array}\right) .
$$

Remark 3.3: Note that by augmenting (12) to (13) and viewing $v^{-}$ as a disturbance input, we obtain a linear discrete time system with set bounded input. While such system can be treated using existing techniques in the literature, less conservative results can be developed by including additional information that $w \in W, w^{-} \in W$.

The augmented system (22) has the form of system (1). In the sequel we use the results of Section III to characterize the MRIA set for the augmented system (20). Our assumptions for system (20) that are related to assumptions 1-3 are now summarized as follows:

Assumption 4: The sets $V$ and $W$ are compact.

Assumption 5: For all $w \in W, g(w)=(F w \oplus G V) \cap W \neq \emptyset$.

Assumption 6: The matrix $A_{L}:=A+B L$ is strictly stable.

Note that Assumption 5 means that the set $V$ is such that for all $w \in W$ there exists $v \in V$ such that $F w+G v \in W$. Morover, Assumption 6 can be easily satisfied if the pair $(A, B)$ is stabilizable, e.g. by any pole placement technique.

\section{Characterization of MRIA Sets: The Special CASE}

In this section, we establish existence of a MRIA set once the disturbance evolves dynamically inside a compact set. To analyze existence and uniqueness of a minimal invariant set for augmented system (22), we consider existence and uniqueness of the fixed point for the mapping

$$
\mathcal{R}(\Omega):=\{\tilde{A} z+w \mid z \in \Omega, \quad w \in \mathcal{W}(z)\}
$$

Proposition 4.1: Suppose Assumptions 4 and 5 hold. Then the correspondence $g$ defined in (17) is upper semi-continuous.

Proof: The Graph of $g$ is

$$
G r g=\{(x, y) \mid x \in W, \quad y \in(F x+G V) \cap W\} .
$$

Since $V$ and $W$ are compact, the sets $G_{1}:=\{(x, y) \mid x \in W, y \in$ $F x+G V\}$ and $G_{2}:=\{(x, y) \mid x \in W, y \in W\}$ are compact. Hence, $G r g=G_{1} \cap G_{2}$ is compact. Therefore, since the range of $g$ is the compact set $W$ and its graph is closed, $g$ is upper semi-continuous [16].

Remark 4.1: We note that even if the sets $V$ and $W$ are compact and convex with zero in their interior, the multifunction $g$ may not be continuous.

Proposition 4.2: Suppose Assumptions 4 and 5 hold. Then the correspondence $\mathcal{W}$, defined in (23), is upper semi-continuous.

Proof: Functions $h_{1}: \mathbb{R}^{n+p} \rightarrow \mathbb{R}^{p}$ given by $h_{1}(z):=\{[0 I] z\}$ and $h_{2}: \mathbb{R}^{p} \rightarrow \mathbb{R}^{n+p}$ given by $h_{2}(z):=\{\tilde{B} z\}$ are continuous and $g$ is upper semi-continuous (according to Proposition 4.1). Therefore, $\mathcal{W}(z)=h_{2} \cdot g \cdot h_{1}$ is upper semi-continuous.

Theorem 4.1: Suppose Assumptions 4-6 hold and

$$
F:=C l\left(\bigoplus_{i=0}^{\infty} \tilde{A}^{i} \tilde{B} W\right) .
$$

Then

$$
\Gamma^{*}:=\bigcap_{j=0}^{\infty} \mathcal{R}^{j}(F)
$$

is non-empty and compact, i.e., $\Gamma^{*} \in \operatorname{Com}\left(\mathbb{R}^{n}\right)$, and $\mathcal{R}\left(\Gamma^{*}\right)=\Gamma^{*}$, with $\mathcal{R}$ defined in (25). Moreover, $\Gamma^{*}$ is robust invariant and is the minimal attractor for the augmented system (22) with the basin of attraction being the whole space, $\operatorname{Com}\left(\mathbb{R}^{n}\right)$. 
Proof: According to Assumption 6, $\tilde{A}$ is stable. Hence, Assumption 1 is satisfied for augmented system (22). According to Assumptions 4 and 5 and Proposition 4.2, $\mathcal{W}$ is upper semi-continuous. So Assumption 2 is satisfied for augmented system (22). Since $g(w) \subseteq W$ for all $w \in W, \mathcal{W}(z) \subseteq \tilde{B} W$ for all $z \in \mathbb{R}^{n} \times W$. Therefore, Assumption 3 is satisfied for augmented system (22). With Assumptions 1-3 satisfied, according to Theorem $2.1, \Gamma^{*}$ is non-empty and is a fixed point of $\mathcal{R}$. Moreover, according to Theorem $2.2, \Gamma^{*}$ is the minimal attractor for the augmented system (22) with the basin of attraction being the whole space. The robust invariance is the immediate result of $\mathcal{R}\left(\Gamma^{*}\right)=\Gamma^{*}$.

We consider the uniqueness of the fixed point $\Gamma^{*}$ of the mapping $\mathcal{R}$ in the sequel.

Lemma 4.1: Suppose Assumptions 4-6 hold and $\Delta \in \operatorname{Com}(W)$ be the fixed point of $g$, i.e. $g(\Delta)=\Delta$. If $\bar{\Gamma}_{n}:=\left\{\sum_{i=0}^{n} \tilde{A}^{n-i} \tilde{B} w_{i} \mid w_{0} \in\right.$ $\left.\Delta, w_{i+1} \in g\left(w_{i}\right), i=0, \cdots, n-1\right\}$, then there exists $\bar{\Gamma} \in \operatorname{Com}\left(\mathbb{R}^{n} \times\right.$ $W$ ) such that

$$
\bar{\Gamma}_{n} \rightarrow \bar{\Gamma} \text {, as } n \rightarrow \infty
$$

in the space $\operatorname{Com}\left(\mathbb{R}^{n} \times W\right)$ with Hausdorff metric.

Proof: Let us define $g^{-1}: \Delta \mapsto \operatorname{Com}(\Delta)$ as follows:

$$
g^{-1}(w):=\{x \in \Delta \mid g(x)=w\} .
$$

Since $g(\Delta)=\Delta, \forall w \in \Delta, g^{-1}(w) \neq \emptyset$. It can be easily shown that

$$
\begin{aligned}
& \mathbb{E}^{n}:=\left\{\left[w_{0}, \cdots, w_{n}\right] \mid w_{0} \in \Delta, w_{i+1} \in g\left(w_{i}\right)\right. \\
&i=0, \cdots, n-1\} \\
&=\{ {\left[w_{0}, \cdots, w_{n}\right] \mid w_{n} \in \Delta, w_{i} \in g^{-1}\left(w_{i+1}\right) } \\
&i=0, \cdots, n-1\} .
\end{aligned}
$$

Therefore $\bar{\Gamma}_{n}=\left\{\sum_{i=0}^{n} \tilde{A}^{i} \tilde{B} w_{i} \mid w_{0} \in \Delta, w_{i+1} \in g^{-1}\left(w_{i}\right), i=\right.$ $0, \cdots, n-1\}$. Since $A_{L}$ is strictly stable, so is $\tilde{A}$. Therefore, the set $F_{\infty}:=\bigoplus_{i=0}^{\infty} \tilde{A}^{i} \tilde{B} \Delta$ is bounded. Hence

$$
\begin{aligned}
& \forall \epsilon>0, \exists N(\epsilon)>0, \text { s.t. } \forall n>N(\epsilon) \\
& \tilde{A}^{n} F_{\infty} \subset B_{\epsilon}(0)=\{w \mid\|w\|<\epsilon\} .
\end{aligned}
$$

Given $\epsilon>0$, and $r>N(\epsilon / 2)$, if $x \in \bar{\Gamma}_{r}$, then

$$
\begin{aligned}
x= & \sum_{i=0}^{r} \tilde{A}^{i} \tilde{B} w_{i} \\
= & \sum_{i=0}^{N} \tilde{A}^{i} \tilde{B} w_{i}+\sum_{i=N+1}^{r} \tilde{A}^{i} \tilde{B} w_{i} \\
& \quad \text { for some }\left[w_{0}, \cdots, w_{r}\right] \in \mathbb{E}^{n} .
\end{aligned}
$$

But $\sum_{i=0}^{N} \tilde{A}^{i} \tilde{B} w_{i} \in \bar{\Gamma}_{N}$ and $\sum_{i=N+1}^{r} \tilde{A}^{i} \tilde{B} w_{i} \in B_{\epsilon / 2}$. Therefore for all $x \in \bar{\Gamma}_{r}, d\left(x, \bar{\Gamma}_{N}\right)<\epsilon / 2$ and hence

$$
\bar{\Gamma}_{r} \subset \bar{\Gamma}_{N} \oplus B_{\epsilon / 2}(0) .
$$

If $x \in \bar{\Gamma}_{N}, \exists\left[w_{0}, \cdots, w_{N}\right] \in \mathbb{E}^{N}$, s.t. $x=\sum_{i=0}^{N} \tilde{A}^{i} \tilde{B} w_{i}$. Choosing $\hat{w}_{1}, \cdots, \hat{w}_{r-N}$ s.t. $\left[\hat{w}_{0}, w_{1}, \cdots, \hat{w}_{r-N}\right] \in \mathbb{E}^{r-\bar{N}}$, and $\hat{w}_{0} \in g\left(w_{N}\right)$, we have $x+\sum_{i=1}^{r-N} \tilde{A}^{N+i} \tilde{B} \hat{w}_{i} \in \bar{\Gamma}_{r}$. Since $\sum_{i=1}^{r-N} \tilde{A}^{N+i} \tilde{B} \hat{w}_{i} \in$ $B_{\epsilon / 2}(0)$, we have $\forall x \in \bar{\Gamma}_{N} d\left(x, \bar{\Gamma}_{r}\right)<\epsilon / 2$, and hence

$$
\bar{\Gamma}_{N} \subset \bar{\Gamma}_{r} \oplus B_{\epsilon / 2}(0) .
$$

From (33) and (34), we have

$$
\forall r>N, \delta_{H}\left(\bar{\Gamma}_{N}, \bar{\Gamma}_{r}\right)<\epsilon / 2 .
$$

From (35), we have

$$
\begin{aligned}
& \forall r_{1}, r_{2}>r \\
& \delta_{H}\left(\bar{\Gamma}_{r_{1}}, \bar{\Gamma}_{r_{2}}\right)<\delta_{H}\left(\bar{\Gamma}_{N}, \bar{\Gamma}_{r_{1}}\right)+\delta_{H}\left(\bar{\Gamma}_{N}, \bar{\Gamma}_{r_{2}}\right) \\
& \quad<\epsilon / 2+\epsilon / 2=\epsilon .
\end{aligned}
$$

Therefore, $\left\{\bar{\Gamma}_{n}: n \in \mathbb{N}\right\}$ is Cauchy and by completeness of Hausdorff metric in $\operatorname{Com}\left(\mathbb{R}^{n} \times W\right), \bar{\Gamma}_{n}$ converges to some $\bar{\Gamma} \in \operatorname{Com}\left(\mathbb{R}^{n} \times W\right)$

Lemma 4.2: Suppose Assumptions 4-5 hold. Then the mapping $g(\cdot)$ has a minimal attracting fixed point $\Delta$, that is

$$
g^{n}(W) \rightarrow \Delta \text { as } n \rightarrow \infty \text {. }
$$

Proof: Proof follows from the fact that $g^{i+1}(W) \subset g^{i}(W)$ and Lemma 2.4.

Theorem 4.2: Suppose Assumptions 4-6 hold. Then

$$
\forall \Lambda \in \mathbb{R}^{n}, \mathcal{R}^{n}(\Lambda \times W) \rightarrow \bar{\Gamma} \text { as } n \rightarrow \infty
$$

where $\bar{\Gamma}$, defined in (28). Moreover, $\bar{\Gamma}=\Gamma^{*}$, defined in (27).

Theorem 4.3: If in (17), $F=G=I$ and $W$ and $V$ are compact, and have zero in the interior, and $W$ is path connected, then $W$ is the unique attracting fixed point of the mapping $g$. Moreover

$$
\forall \Lambda \in \operatorname{Com}(W), g^{n}(\Lambda) \rightarrow W \text {, as } n \rightarrow \infty \text {. }
$$

See [18] for the proof of the above Theorems.

Remark 4.2: We note that if $W$ and $V$ are compact, convex, and zero in their interior and the matrix $F$ is strictly stable, the mapping $g$ may not have a unique fixed point. Consider the sets

$$
\begin{aligned}
W & =\left\{x \in \mathbb{R}^{2}||[41] x|\leq 4,|[4-1] x \mid \leq 4\right\} \\
V & =\left[\begin{array}{ll}
-1 & 1]
\end{array}\right] \operatorname{Com}(\mathbb{R})
\end{aligned}
$$

with $F=\left[\begin{array}{cc}0 & -0.5 \\ 0.5 & 0\end{array}\right]$ and $G=\left[\begin{array}{ll}2 & 4\end{array}\right]^{T}$. It can be checked that the sets $W$ and $\left\{\left[\begin{array}{ll}0 & 4\end{array}\right]^{T}\right\}$ are fixed points of the mapping $g$ defined in (17). Therefore, the mapping $g$ may not have a unique fixed point even if $W$ and $V$ are compact and convex and have zero in their interior and $F$ is strictly stable.

Remark 4.3: We note that the results of this section hold as long as $A_{L}$ is strictly stable, independent of the choice of the Matrix $M$. However, the matrix $M$ contributes in the size of the minimal attractor $\bar{\Gamma}$.

\section{NUMERICAL EXAMPLE}

In this section, we demonstrate the effect of including the rate bound of the disturbance on the MRIA set. We consider roll dynamics of a ship equipped with fin stabilizers [19]. The following linear equations describe the roll motion expressed in a body fixed frame with the origin at the center of gravity of the ship:

$$
\dot{\phi}=p, I_{\phi \phi} \dot{p}+D p+G \phi=\tau_{c}+\tau_{w}
$$

where $\phi$ is the roll angle, $p$ is the roll rate, $\tau_{c}$ is the control moment produced by the fins, and $\tau_{w}$ is the wave excitation moment. Moreover, $I_{\phi \phi}$ is the total inertia in roll about the axis along the ship longitudinal direction, $D$ is the equivalent linear damping (which accounts for potential and viscous effects), and $\mathrm{G}$ is the linear roll restoring coefficient.

Given the ship model and coefficients provided in [19], the discretetime model of (40), with sampling period $T_{s}=0.1 \mathrm{sec}$, is

$$
x^{+}=A_{d} x+B_{d} u+B_{w} w
$$




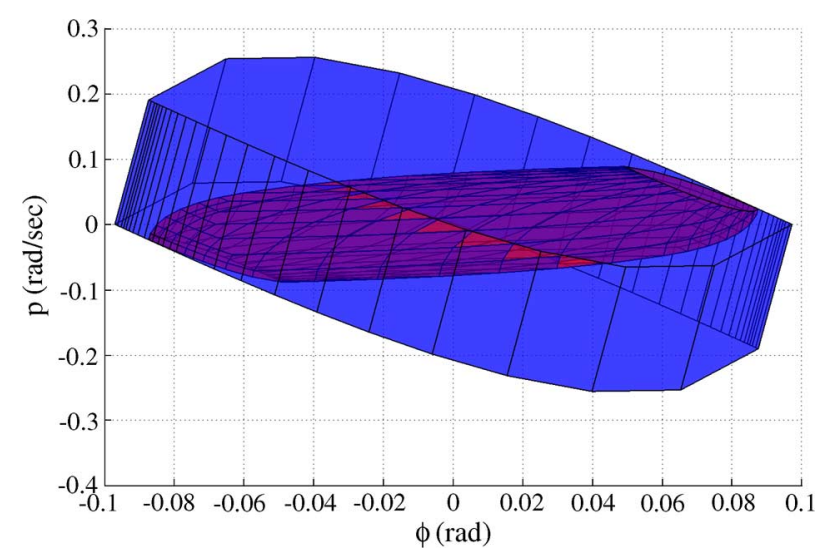

Fig. 1. MRIA sets for $r_{w}=\infty$ (blue) and $r_{w}=0.1$ (red), viewed from above (third axis is not shown).

where $x=[\phi p]^{T}, u=\tau_{c}, w: \tau_{w} / I_{\phi \phi}$ is the normalized wave moment with $|w| \leq 1$ which acts as unmeasured disturbance, and

$$
A_{d}=\left[\begin{array}{cc}
0.99 & 0.095 \\
-0.08 & 0.90
\end{array}\right], B_{d}=\left[\begin{array}{l}
-0.007 \\
-0.142
\end{array}\right], B_{w}=\left[\begin{array}{l}
0.004 \\
0.095
\end{array}\right] .
$$

The feedback gain $K=[-6.31-3.66]$ is designed using LQR techniques with weight $R=10$ for control input and the weight $Q=$ $\operatorname{diag}[102]$ for the states. The MRIA set for the system

$$
x^{+}=\left(A_{d}+K B_{d}\right) x+B_{w} w
$$

plays a pivotal role in the design of constrained robust controllers [1], [4]. Practically, the wave moment does not vary arbitrarily and is both magnitude and rate bounded. We consider two cases where the normalized rate bound on the wave moment, i.e. $r_{w}:=w^{+}-w$, is $\infty$ (i.e., we only model the magnitude limits) and 0.1 , respectively. Fig. 1 shows the MRIA sets corresponding to the two rates in the lifted space, viewed from above. Due to point of view, it also shows the projections of MRIA sets onto $\mathcal{R}^{2}$ which are MRIA sets in the state space of the system (40). Clearly, in the lifted space the MRIA set is considerably smaller once the rate-boundedness of the disturbance $w$ is considered. Moreover, the MRIA set for system (40), i.e. projection of MRIA set from lifted space onto $\mathcal{R}^{2}$, is appreciably larger if the rate-boundedness is neglected. The details of the computation of the MRIA set are discussed in [20].

\section{CONCLUSION}

In this technical note, we examined the attractiveness and minimality of invariant sets for linear systems subject to disturbances confined in state-dependent sets. Assuming upper-semi-continuity and boundedness of the correspondence associated with additive disturbance, we proved existence and uniqueness of a minimal attractor set, which is robust invariant. In the case where the dynamics of the disturbance are known, the results were used to characterize a minimal attractor set. Our subsequent work will exploit the application of these ideas to MPC design.

\section{ACKNOWLEDGMENT}

The authors would like to thank Dr. S. V. Rakovic for his help, constructive comments, and suggestion of several steps in this development.

\section{REFERENCES}

[1] D. Q. Mayne, M. M. Seron, and S. V. Rakovic, "Robust model predictive control of constrained linear systems with bounded disturbances," Automatica, vol. 41, no. 2, pp. 1136-1142, 2005.

[2] F. Blanchini and S. Miani, Set-Theoretic Methods in Control. Boston, MA: Birkhauser, 2008.

[3] A. Bemporad, A. Casavola, and E. Mosca, "Nonlinear control of constrained linear systems via predictive reference management," IEEE Trans. Autom. Control, vol. 42, no. 3, pp. 340-349, Mar. 1997.

[4] E. G. Gilbert and I. Kolmanovsky, "Fast reference governors for systems withstate and control constraints and disturbance inputs," Int. J. Robust Nonlin. Control, vol. 9, no. 15, pp. 1117-1141, Dec. 1999.

[5] D. Q. Mayne, J. B. Rawlings, C. V. Rao, and P. O. M. Scokaert, "Constrained model predictive control: Stability and optimality," Automatica, vol. 36, pp. 789-814, 2000.

[6] D. L. Marruedo, T. Alamo, and E. F. Camacho, "Stability analysis of systems with bounded additive uncertainties based on invariant sets: Stability and feasibility of MPC," in Proc. Amer. Control Conf., 2002, pp. 364-369.

[7] I. Kolmanovsky and E. Gilbert, "Theory and computation of disturbance invariant sets for discrete-time linear systems," Math. Problems Eng.: Theory, Methods Appl., vol. 4, pp. 317-367, 1998.

[8] S. V. Raković and M. Fiacchini, "Invariant approximations of the maximal invariant set or "Encircling the Square"," in Proc. 17th IFAC World Congress IFAC'08, Seoul, Korea, Jul. 2008, pp. 6377-6382.

[9] L. Chisci, J. A. Rossiter, and G. Zappa, "Systems with persistent disturbances: Predictive control with restrictive constraints," Automatica, vol. 37, pp. 1019-1028, 2001.

[10] Z. Artstein and S. Raković, "Feedback and invariance under uncertainty via set-iterates," Automatica, vol. 44, pp. 520-525, 2007.

[11] E. Kofman, H. Haimovich, and M. M. Seron, "A systematic method to obtain ultimate bounds for perturbed systems," Int. J. Control, vol. 80, no. 2, pp. 167-178, 2007.

[12] I. Komanovsky, E. G. Gilbert, and N. H. McClamroch, "Towards less conservative design of multimode controllers for systems with state and control constraints," in Proc. Conf. Decision Control, Tampa, FL, 1998, pp. 1647-1652.

[13] S. V. Rakovic, "Minkowski algebra and banach contraction principle in set invariance for linear discrete time systems," in Proc. 46th IEEE Conf. Decision Control, 2007, pp. 2169-2174.

[14] M. Milanese and A. Vicino, "Optimal estimation theory for dynamic systems with set membership uncertainty: An overview," Automatica, vol. 27, no. 6, pp. 997-1009, 1991

[15] E. Klein and A. C. Thompson, Theory of Correspondences. New York: Wiley-Interscience, 1984.

[16] C. D. Aliprantis and K. C. Border, Infinite Dimentional Analysis. New York: Springer, 2005.

[17] J. L. Kelley, General Topology. New York: Springer, 1975.

[18] R. Ghaemi, "Robust Model Based Control of Constrained Systems," Ph.D. dissertation, Univ. Michigan, Ann Arbor, 2010.

[19] R. Ghaemi, J. Sun, and I. V. Kolmanovsky, "Robust control of ship fin stabilizers subject to disturbances and constraints," in Proc. Amer. Control Conf., St. Louis, MO, 2009, pp. 537-542.

[20] R. Ghaemi, I. V. Kolmanovsky, and J. Sun, "Robust control of linear systems with bounded state dependent additive disturbances," in Proc. IEEE Conf. Decision Control, Atlanta, GA, 2010, pp. 5198-5203. 